\title{
IMPACT OF MATERIALS ON BACK-CONTACT MODULE RELIABILITY
}

\author{
M.Guichoux, C.Tjengdrawira, D.Veldman, P.C. de Jong \\ Energy research Centre of the Netherlands, Solar Energy, P.O. Box 1, NL-1755 ZG Petten, the Netherlands \\ Phone: +31 22456 4875; Fax: +31 22456 8214; email: guichoux@ecn.nl
}

\begin{abstract}
This paper discusses the impact of several combinations of encapsulants, conductive adhesives and back-sheet foils on ECN's back-contact modules performance and reliability. Damp-heat $\left(85^{\circ} \mathrm{C} ; 85 \%\right.$ R.H) and thermal cycling $\left(-40^{\circ} \mathrm{C} ;+85^{\circ} \mathrm{C}\right)$ tests were performed up to twice as long as described in the IEC-61215 standard, i.e. 2000 hours of damp-heat and 400 thermal cycles. These tests have been identified as the most important tests for this technology. After climatic chamber testing, the modules were analyzed visually and by electro-luminescence imaging.
\end{abstract}

\section{INTRODUCTION}

ECN has developed and demonstrated a single-step module encapsulation and interconnection technology for Metallization-Wrap-Through (MWT) solar cells [1] [2]. This work lead to the manufacturing of the world's first multicrystalline silicon module to reach $17.0 \%$ conversion efficiency [3]. The interconnection process is based on the use of a conductive adhesive which drains the current from the MWT cells to a conductive backsheet foil underneath. It is essential to ensure a reliable structure throughout service life. The sensitivity of such construction to climatic variations is likely to be different from conventional module constructions. Therefore, a test program has been set-up to investigate climatic-chamber induced degradation effects, such as mechanical stresses and/or chemical reactions, which lead to performance losses.

\section{APPROACH}

Thirty-six four-cell mini-modules were assembled according to the process described in [4]:

- stencil printing of conductive adhesive spots on the contact areas of the backsheet foil;

- $\quad$ placement of a pre-punched sheet of encapsulant sheet;

- alignment and placement of the MWT cells so that the cells' contacts match the printed conductive adhesives spots;

- $\quad$ placement of the top encapsulant sheet;

- $\quad$ placement of the cover glass sheet.

Subsequently, the package is laminated into a solar minimodule.

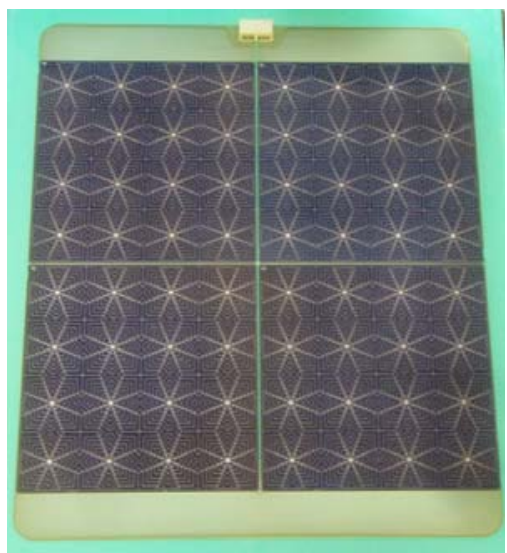

Figure1: Front view of an MWT mini-module [5].

These mini-modules were subjected to damp-heat and thermal cycling durability tests and split in nine different combinations of materials and test methods. The thermal cycling tests were carried out with a forward bias current of 7 Amperes. The materials used to fabricate these modules included two types of backsheet foil supplier (1, 2), two types of encapsulants (A,B) and three types of conductive adhesives (i, ii, iii)

\begin{tabular}{|c|c|c|c|c|}
\hline Group & Test & Backsheet foil & Encapsulant & C.A \\
\hline 1 & $\mathrm{DH} 2000$ & 1 & $\mathrm{~A}$ & $\mathrm{i}$ \\
\hline 2 & $\mathrm{DH} 2000$ & 2 & $\mathrm{~A}$ & $\mathrm{i}$ \\
\hline 3 & $\mathrm{DH} 2000$ & 2 & $\mathrm{~B}$ & $\mathrm{i}$ \\
\hline 4 & $\mathrm{DH} 2000$ & 2 & $\mathrm{~B}$ & $\mathrm{ii}$ \\
\hline 5 & $\mathrm{DH} 2000$ & 2 & $\mathrm{~B}$ & $\mathrm{iii}$ \\
\hline 6 & $\mathrm{TC} 400$ & 2 & $\mathrm{~B}$ & $\mathrm{iii}$ \\
\hline 7 & $\mathrm{TC} 400$ & 2 & $\mathrm{~A}$ & $\mathrm{iii}$ \\
\hline 8 & $\mathrm{DH} 2000$ & 1 & $\mathrm{~A}$ & $\mathrm{iii}$ \\
\hline 9 & $\mathrm{DH} 2000$ & 2 & $\mathrm{~A}$ & $\mathrm{iii}$ \\
\hline
\end{tabular}

Table 1: Overview of the modules manufactured; each group is made of four identical modules

Current-voltage characteristics were measured after every 500 hours of damp-heat testing or every 100 thermal cycles, using a class-A flash-tester. Following the climatic tests, the modules were first analyzed visually and then by electro-luminescence (E.L) imaging.

Using four-cell modules is a logical step for materials qualification in the up scaling process from coupons or single-cell modules to industrial-size modules. It is important to note that the four-cell modules were not sealed or framed. Thus, they were more severely exposed than edge-sealed and framed full-size modules. Furthermore, using mini-modules allows increasing the 
sample size, which is critical to statistically analyze the outcome of the experiments.

\section{RESULTS}

\subsection{Relative output power (I-V)}

The mini-modules were tested and characterized against criteria as set out by the IEC-61215 requirements, i.e., the module should have kept at least $95 \%$ of its initial output power.

Figure 2 shows an overview of output power degradation of all the modules during damp-heat (blue) and thermal cycling (red) testing. After 1xIEC testing, over $94 \%$ $(32 / 36)$ of the thirty-six mini-modules are still delivering $95 \%$ or more of their initial output power. However, after $2 x I E C$ testing, this percentage has dropped to $72 \%$ (26/36). Damp-heat testing appeared to be the most critical test as none of the modules tested in thermal cycling suffers a $\mathrm{P}_{\max }$ decrease higher than 1\%. More detailed results, averaged per group of four modules are displayed in Table 1. As thermal cycling appeared not to be problematical, the main focus is on the groups tested under damp-heat conditions.

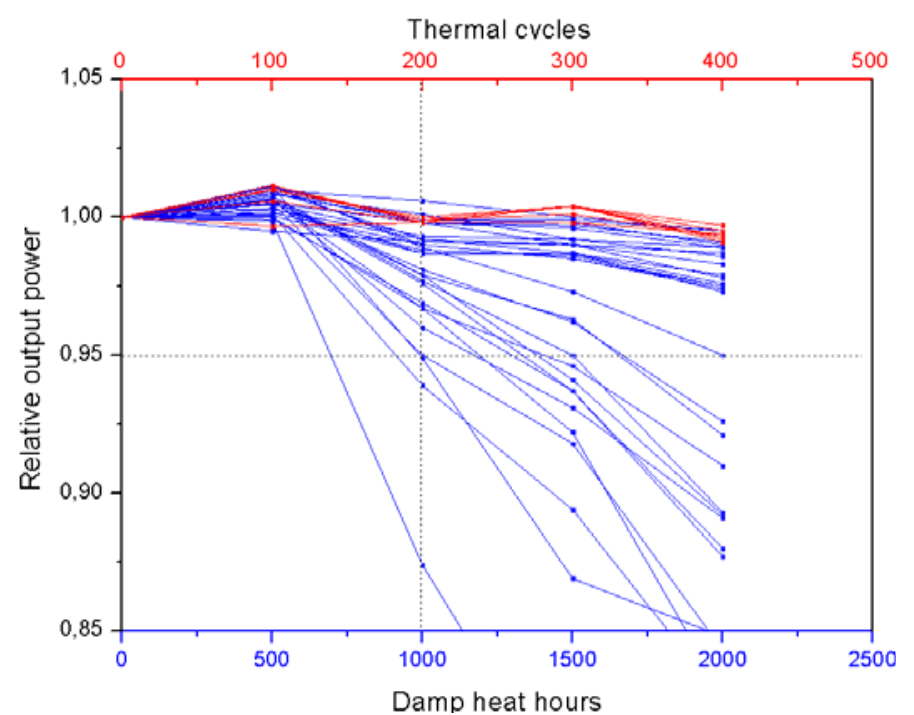

Figure 2: Overview of the power output degradation of the $\mathbf{3 6}$ modules as a function of damp-heat (blue lines) and thermal cycling (red lines).

First, it is important to note that, on average, all the material combinations described in Section 2 passed the $95 \%$ criterion after a standard IEC-like testing: 1000 hours of damp-heat or 200 thermal cycles (1xIEC). It is only when doubling the test sequence (2xIEC) that some dropped below $95 \%$ relative output power.

\begin{tabular}{|c|r|r|}
\hline Group & \multicolumn{1}{|c|}{ 1xIEC } & 2xIEC \\
\hline 1 & $96.1 \%$ & $87.3 \%$ \\
\hline 2 & $96.3 \%$ & $86.3 \%$ \\
\hline 3 & $99.2 \%$ & $97.6 \%$ \\
\hline 4 & $99.5 \%$ & $98.9 \%$ \\
\hline 5 & $99.9 \%$ & $99.1 \%$ \\
\hline 6 & $100.0 \%$ & $99.4 \%$ \\
\hline 7 & $100.0 \%$ & $99.3 \%$ \\
\hline 8 & $95.4 \%$ & $91.4 \%$ \\
\hline 9 & $99.1 \%$ & $97.8 \%$ \\
\hline
\end{tabular}

Table 2: Relative output power for each group described in Table 1 after 1 x IEC and 2 x IEC climatic testing. Each data point is an average of four modules

$>$ Backsheet foil supplier: all of the 8 modules built with material from supplier 1 - groups 1 and 8 - degraded more than $5 \%$ after 2000 hours of damp-heat testing. After 1000 hours, these were already more severely degraded (relative output power $=96.1 \%$ and $95.4 \%$ respectively). Supplier 2's material is clearly performing better as numerous modules manufactured with it survived the extended 2xIEC test. To consistently compare encapsulants and conductive adhesives, groups 1 and 8 have been discarded in the following sub-sections.

$>$ Encapsulant: all the failing modules - groups 1, 2 and 8 - were encapsulated with material $A$ while all the modules built with encapsulant B passed. Modules from groups 2 and 9 can be respectively compared to those from groups 3 and 5, as they are identical except for the encapsulants. In both cases, modules encapsulated with material B perform better: $86.3 \%$ versus $97.6 \%$ and $97.8 \%$ versus $99.1 \%$, respectively, of relative output power after 2000 hours of damp-heat testing. Conductive adhesives comparison (see below) should then be also distinguished with respect to the encapsulant used. Material $\mathrm{B}$ would be the recommended choice for further experiment.

$>$ Conductive adhesive: when using encapsulant A, conductive adhesive iii provides a more reliable interconnection than i; indeed groups 2 and 9, which are identical except for the conductive adhesive ( $i$ versus iii) respectively conserve a relative output power of $86.3 \%$ and $97.8 \%$. On the other hand, when using encapsulant $\mathrm{B}$, there is no significant difference when comparing based on conductive adhesive choice. All the modules retain about $99 \%$ of their initial output power (groups 3, 4 and 5). Thus, with encapsulant A, conductive adhesive iii is preferred while with encapsulant $B$ the choice of the conductive adhesive is of less importance as all of them performed very well.

Finally, based on these data, mini-modules having lost less than $1 \%$ of their power after $1 \times$ IEC typically survive $2 \times$ IEC testing. 


\subsection{Visual inspection}

Visual inspection of the modules after climatic chamber testing revealed no major issues. Neither backsheet foil suppliers nor conductive adhesive types have an influence on the appearance of the modules. However, the encapsulant choice is a determining factor. Modules manufactured with encapsulant B are electrically performing very well, but show numerous localized dark brown spots outside the cell area, as shown in Figure 4. Modules encapsulated with material $A$ do not suffer from inhomogenity although they do appear darker after testing. Additionally, one sample suffered from visible delaminations. This module is catastrophically degraded between 500 hours and 1000 hours of damp heat testing (relative output power $=88 \%$ after 1000 hours).

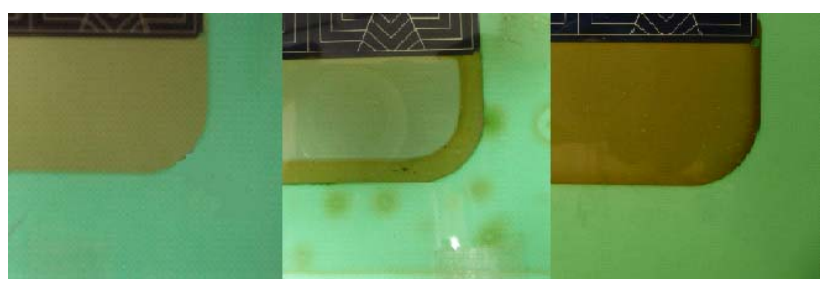

(a)

(b)

(c)

Figure 4: Close-up of a corner section of the minimodules, (a) after lamination, (b) after 2000 hours of damp-heat with encapsulant $B$ (middle) and (c) after 2000 hours of damp-heat testing with encapsulant $A$ (right)

The differences in the encapsulants' appearance after damp-heat testing are attributed to interactions with the conductive backsheet foils. Some encapsulants are known to degrade when exposed to heat and/or humidity [6]. The products of their degradation can be harmful for the PV materials they are in contact with. ECN's back-contact module technology uses a new type of conductive backsheet foil for which interaction mechanism(s) with encapsulants and their product of degradation is part of daily research. Areas of further investigation are to study the effects of differences in moisture permeability between types of encapsulant.

\subsection{Electro-Luminescence (E.L.)}

After completion of the climatic testing, the modules were analyzed by electro-luminescence, with a particular focus on the ones severely degraded. This aimed at gaining an understanding of the failure mechanism(s) responsible for the power decrease. It is possible to create an image of the local series resistance variations in the module. This can be used to detect degraded or failing interconnections. [7]. Figure 5 shows the E.L. images of two severely degraded modules. The red areas correspond to increased series resistance, so inactive areas. On the left image a high series resistance is visible around several emitter (front side) contacts while on the right image, the series resistance is increased at the exact location of the base (rear side) contacts. In both cases the E.L. image helps detecting the degraded contacts which facilitates further (destructive or not) post-test analysis. The difference mentioned between modules presenting either degraded emitter contacts or degraded base contacts suggests that the MWT cells can also be involved in the failure mechanism because, from a module and conductive backsheet foil perspective, emitter and base contacts are identical.
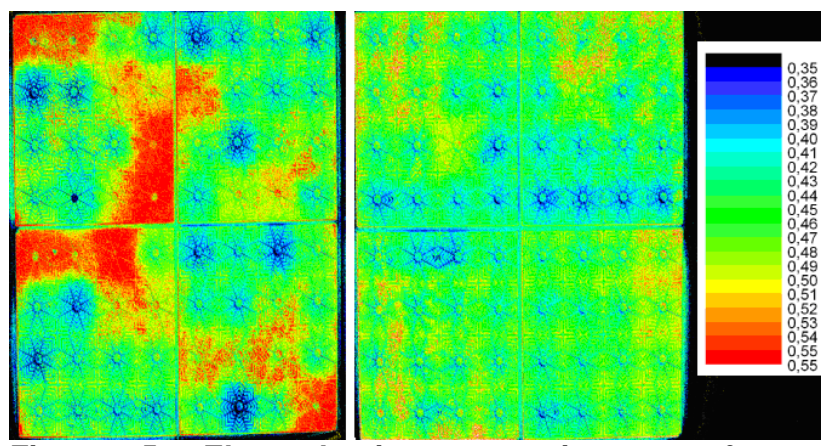

Figure 5: Electro-luminescence images of two degraded modules $(82.7 \%$ and $84.8 \%$ of relative output power). Degraded emitter (left) and base (right) contacts are visible

\subsection{Delamination}

After non-destructive analysis, modules have been disected for further analysis. The existence of delamination areas was further investigated and a rootcause analysis was set-up. A key finding is that the quality of the isolation coating is extremely important as it acts as an in-between layer between encapsulant and metal conductor layer. Material interaction between encapsulant and isolation coating appears to be the root cause of delamination.

So far no solid correlation between delamination and degraded interconnections visible in E.L has been demonstrated. However, if the conductive backsheet foil delaminates from the encapsulant, the cohesion of the complete structure will only be maintained by the conductive adhesive which will then suffer increased mechanical stress. This could lead to a breakage of the current conduction path, either at the adhesive/foil interface, or at the adhesive/cell interface, or cohesively within the conductive adhesive. Interconnection zones are amongst the most sensitive of this type of back-contact modules because they regroup, on one location, all the critical components: cell, encapsulant, conductive adhesive and conductive backsheet foil. 


\section{CONCLUSIONS}

We have shown that numerous MWT four-cell modules manufactured at ECN successfully passed extended climatic testing (2000 hours of damp-heat, 400 thermal cycles). Electro-luminescence is confirmed to be an interesting tool to locate degraded interconnections in MWT modules. Special attention has to be paid when it comes to materials selection. The results presented in this paper demonstrate that ECN's back-contact module technology with conductive adhesives and a conductive foil as interconnection is reliable enough to withstand stringent climatic tests and is ready to be further tested on full-size modules and later for module certification.

\section{ACKNOWLEDGEMENTS}

The authors want to thank Q.Cells for manufacturing the MWT solar cells. This work was carried out in projects with financial support from the Dutch agency AgentschapNL.

\section{REFERENCES}

[1] P.C. de Jong et al., "Single-step laminated full size PV modules made with back-contacted mc-Si cells and conductive adhesives", $19^{\text {th }}$ European Photovoltaic Solar Energy Conference and Exhibition, Paris (2004).

[2] P.C. de Jong et al., "Beyond 16\% efficient back-contact modules using Mc-Si wafers and conductive adhesive interconnection technology", $22^{\text {nd }}$ European Photovoltaic Solar Energy Conference and Exhibition, Milan (2007).

[3] C. Tjengdrawira et al., "World first module with $17 \%$ efficiciency using multicrystalline silicon solar cells", this conference.

[4] M.Spath et al., "A novel module assembly line using back contact solar cells", IEEE Photovoltaic Specialists Conference, San Diego (2008).

[5] Solland Solar Energy Holding BV is the owner of the registered industrial designs Sunweb ${ }$.

[6] A.W. Czanderna and F.J Pern, "Encapsulation of PV modules using ethylene vinyl acetate copolymer as a pottant: A critical review", Solar Energy Materials and Solar Cells 43 (1996) 101-181.

[7] U. Hoyer et al., "Electroluminescence and infrared imaging for quality improvements of PV modules", $23^{\text {rd }}$ European Photovoltaic Solar Energy Conference and Exhibition, Valencia (2008). 